\title{
Diagnosis of Primary Cancer of the Liver
}

\author{
M. C. KEW, H. A. DOS SANTOS, SHEILA SHERLOCK
}

British Medical fournal, 1971, 4, 408-411

\section{Summary}

The diagnosis of primary cancer of the liver was reviewed in 75 patients. A definitive diagnosis was made during life in $63 \%$ and in a further $20 \%$ this condition was suspected though histological confirmation was obtained only at necropsy. The most common presenting complaints were abdominal pain and weight loss and the most frequent findings hepatomegaly and ascites. Less than one-half of the patients were jaundiced and when present it was usually mild. An arterial bruit was heard over the liver in $25 \%$ of the patients. $A$ sudden and unexplained deterioration in a patient known to have cirrhosis or haemochromatosis should raise the possibility of a primary hepatic tumour; this occurred in $24 \%$ of our patients.

Alpha-fetoprotein was found in the serum of 11 out of 18 cases. The presence of a mass in the liver was frequently confirmed by liver scan, portal venography, or hepatic arteriography, but these showed no features diagnostic of a primary tumour. Liver scan also proved useful in localizing the lesion for biopsy purposes. Definitive diagnosis is dependent on the histological demonstration of the features of the tumour. This can frequently be achieved by percutaneous needle biopsy, which was positive in 38 out of 57 patients. Wedge biopsies were positive in a further nine patients.

Department of Medicine, Royal Free Hospital, London WC1X 8LF M. C. KEW, M.D., M.R.C.P., Honorary Clinical Assistant and Wellcome Research Fellow

H. A. DOS SANTOS, M.D., Honorary Research Fellow

S. SHERLOCK, M.D., F.R.C.P.(ENG., ED.), Professor of Medicine

\section{Introduction}

The diagnosis of primary cancer of the liver is usually made late in the course of the disease and frequently only at necropsy. There are many reasons for this. Because of the large size of the liver the tumour usually has to reach a considerable size before it can be felt or before it invades adjacent structures. The liver is relatively inaccessible to the examining hand. The hepatic reserves are such that a large proportion of the liver must be replaced before jaundice or other signs of liver failure appear. Distant metastases are uncommon and usually appear late in the course of the disease. ${ }^{12}$ There are no specific biochemical changes ${ }^{3}$ and, finally, it is a rare tumour in most parts of the world ${ }^{5-7}$ and almost invariably runs a short course.

The discovery by Tatarinov in $1964^{8}$ of a fetal alpha ${ }_{1}$-globulin in the serum of patients with primary cancer of the liver gave promise of a means to make an early diagnosis and thus, perhaps, to improve the dismal results of treatment. It soon became apparent, however, that the frequency with which alphafetoprotein was found in the serum of these patients showed a striking geographical variation and that false-negative results occurred in as many as $72 \%$ of the patients in some series. ${ }^{9}$ More recently occasional false-positive results have also been described.10-16 Thus though the finding of alpha-fetoprotein in the serum points strongly to the diagnosis of primary hepatic cancer definitive diagnosis still depends on the histological demonstration of the typical features of the tumour. The disease is less likely to be recognized during life in those parts of the world where the tumour is uncommon and the alphafetoprotein test frequently negative. Nevertheless, with a greater awareness of the condition and the use of newer diagnostic techniques it should be possible to make this diagnosis in most instances.

\section{Patients and Methods}

We have reviewed our experience in the diagnosis of this tumour in 75 patients with histologically proved primary cancer 
of the liver seen at the Royal Free Hospital between 1960 and 1971. There were 66 males and 9 females. Their ages ranged from 9 to 75 years but most presented in the fifth, sixth, and seventh decades (Table I). Fifty-nine of the subjects were Caucasian (of whom 45 were born and lived in Britain), while the remainder came from Africa (6), India (5), the Middle East (3), and the West Indies (2). A history of excessive alcohol

taBle I-Age Distribution of the 75 Patients

\begin{tabular}{ll|c|c|c|c|c|c|c|c}
\hline $\begin{array}{l}\text { Age in years } \\
\text { No. of patients }\end{array}$ & $\cdots$ & $\leqslant 10$ & $11-$ & $21-$ & $31-$ & $41-$ & $51-$ & $61-$ & $71-80$ \\
\hline
\end{tabular}

consumption was obtained in 14 patients, nine had a previous attack of acute hepatitis, and a further eight had been jaundiced without a definite cause being established. Twenty-five of the patients were known to have cirrhosis of the liver and two haemochromatosis (idiopathic in one and associated with congenital spherocytosis and multiple blood transfusions in the other) before the presenting illness.

The serum bilirubin, alkaline phosphatase, aspartate aminotransferase, cholesterol, and albumin levels were recorded in each patient, as was the haemoglobin and leucocyte count. Alpha-fetoprotein was looked for in the serum by double diffusion in agar gel (micro-Ouchterlony technique). Hepatitisassociated antigen was detected by using the Ouchterlony gel diffusion technique and counter electrophoresis. Hepatic scintiscans were performed with either colloidal gold-198 or technetium-99m sulphide or indium-113m with a Picker Magnascanner V or a Nuclear Enterprises Scinticamera III. In one patient L-selenomethionine ${ }^{75} \mathrm{Se}$ was used. Selective coeliac axis and superior mesenteric arteriography and splenic venography was performed in the standard way.

\section{Results}

\section{FREQUENCY OF DIAGNOSIS}

A definitive diagnosis of primary hepatic cancer was made during life in 47 patients $(63 \%)$. All but eight of these were suspected of having this condition before the percutaneous biopsy or laparotomy at which the diagnosis was confirmed. The exceptions had an enlarged liver of uncertain cause or, in two cases, presented as an acute abdominal emergency and the diagnosis was made at the ensuing laparotomy; in one instance a primary tumour was discovered at the time of portacaval anastomosis for bleeding from oesophageal varices. A primary hepatic tumour was strongly suspected in a further 15 patients but histological confirmation was obtained only at necropsy. In 11 of these a needle biopsy had been negative. In the remaining 13 patients $(17 \%)$ a tumour not suspected clinically was found at necropsy. Of the latter six were known to have and three were correctly suspected of having cirrhosis, and their deterioration was attributed to this; the symptoms and signs of the tumour were incorrectly attributed to cirrhosis in one patient and to non-hepatic disease in the remaining two.

The mean time elapsing between the onset of symptoms and the diagnosis was four months (range less than 1 month to 10 months). The mean total duration of disease from the onset of symptoms to death was six months (range less than 1 month to 34 months).

\section{CLINICAL FEATURES}

The most common presenting complaints were abdominal pain or discomfort and weight loss (Table II). Pain was usually constant and ill-defined and was severe in only five instances.
TABLB II-Main Symptoms and Physical Signs in the 75 Patients

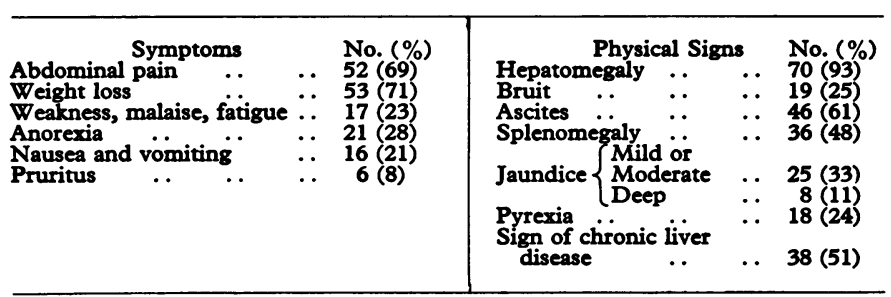

It was usually felt in the right hypochondrium (22 cases) or epigastrium ( 21 cases) but was generalized in nine patients. In five abdominal pain was accompanied by pain in the right shoulder and in two by pain in the back. A further four patients complained only of right shoulder pain and one only of backache. Five patients were aware of a swelling or had a feeling of fullness in the upper abdomen. Two patients were admitted in a shocked state with the features of an acute abdominal crisis. At laparotomy in both cases the peritoneal cavity contained a large quantity of blood and a vessel on the surface of the liver was seen to be eroded by an underlying tumour. One patient was asymptomatic; an enlarged liver (with a bruit) was discovered during a routine medical examination.

On admission to hospital the general condition of 18 patients was poor, it was fair in 37, and good in 20 . In only five patients was the liver not enlarged, and three of these had tense ascites at the time of the examination. The surface of the liver was obviously irregular in 18 instances and a definite mass could be felt in a further eight. An arterial bruit was heard over the enlarged liver in 19 patients $(25 \%)$. In one instance the bruit disappeared after a successful partial hepatectomy. In one further patient a friction rub was heard over the liver. When present jaundice was usually mild; only eight subjects were deeply jaundiced and these were seen late in the course of the disease. The ascitic fluid was examined in 34 of the 46 patients with ascites. It was blood-stained in eight and the protein content exceeded $3 \mathrm{~g} / 100 \mathrm{ml}$ in only 3 out of 14 cases.

A persistent mild or moderate pyrexia was present in 18 patients, six of whom also had a neutrophil leucocytosis. Of the subjects showing signs of chronic liver disease (spider naevi, palmar erythema, etc.) 31 were shown to have hepatic cirrhosis. The presence of a primary hepatic tumour was suspected in 18 patients $(24 \%)$ known to have cirrhosis or haemochromatosis because of a sudden and unexplained deterioration in their clinical condition. Their usual complaints were abdominal pain, weight loss, and swelling of the abdomen, and suggestive signs a sudden enlargement of or development of a mass in the liver, an arterial bruit heard over the liver, or the finding of blood-stained ascites.

\section{BIOCHEMICAL AND IMMUNOLOGICAL FINDINGS}

The alkaline phosphatase was frequently higher than would be expected in a patient with uncomplicated cirrhosis of the liver.

TABLE III-Summary of Relevant Biochemical Findings in the 75 Patients

\begin{tabular}{|c|c|c|c|c|c|}
\hline & \multicolumn{2}{|c|}{ Normal } & \multicolumn{3}{|c|}{ Abnormal } \\
\hline & $\begin{array}{c}\text { No. of } \\
\text { Patients }\end{array}$ & Reading & $\begin{array}{l}\text { No. (\%) of } \\
\text { Patients }\end{array}$ & Range & Mean \\
\hline \multirow{7}{*}{$\begin{array}{l}\text { Bilirubin (mg/100 ml) } \\
\text { Alkaline phosphatase } \\
\text { (K.A. units) . } \\
\text { Aspartate aminotrans- } \\
\text { ferase (units) } \\
\text { Albumin (g/100 ml) } \\
\text { Cholesterol (mg/100 } \\
\text { ml) } \\
\text { Haemoglobin (g/100 } \\
\text { ml) } \\
\text { Leucocyte count (No./ } \\
\text { mm }^{2} \text { ) .. }\end{array}$} & 25 & $<1 \cdot 0$ & $49(66)$ & $1 \cdot 5-40$ & 6.5 \\
\hline & 10 & $<13$ & $63(86)$ & $15-160$ & 36 \\
\hline & 13 & $<17$ & $59(82)$ & $18-770$ & 58 \\
\hline & 26 & $>3.5$ & $47(64)$ & $1 \cdot 7-3 \cdot 4$ & $2 \cdot 1$ \\
\hline & 34 & $<260$ & $12(26)$ & $280-500$ & 380 \\
\hline & 11 & & $63(85)$ & & 11.2 \\
\hline & 59 & & $15(20)$ & $11,500-23,300$ & 14,500 \\
\hline
\end{tabular}


A significantly raised serum alkaline phosphatase concentration ( $>20$ K.A. units) with a normal or only slightly raised serum bilirubin level, suggestive of a space-occupying or infiltrative lesion in the liver, was found in 24 patients $(32 \%)$. Twenty-five subjects $(33 \%)$ had a normal serum bilirubin concentration at the times they were seen. In only eight instances was the serum bilirubin level greater than $10 \mathrm{mg} / 100 \mathrm{ml}$. Three patients suffered hypoglycaemic attacks.

Alpha-fetoprotein was found in the serum of 11 out of 18 patients. Of these five were from Britain, two from Greece, two from Africa, and one each from the Middle East and the West Indies. Results in five out of nine British patients were positive. Hepatitis-associated antigen was present in the serum of 4 out of 12 patients. Three of the positive patients were also positive for alpha-fetoprotein, as were three of the patients in whom hepatitis-associated antigen was not detected.

\section{ISOTOPIC SCANNING OF THE LIVER}

Hepatic scintiscans were performed in 34 patients. Definite evidence of one or more filling defects in the liver was found in 30 instances. In seven of these there was also a patchy loss of uptake of the isotope throughout the liver or splenic uptake suggestive of underlying cirrhosis, though the former could also have been due to multiple small areas of malignancy throughout the liver. In a further two patients there were areas of decreased uptake of the isotope suggestive but not diagnostic of replacement of hepatic tissue, and in the final two only a patchy loss of uptake thought to be due to cirrhosis. There were no features on the positive scans which distinguished between primary hepatic cancer and other space-occupying lesions in the liver. In the only patient studied in this way, however, the filling defect seen on a technetium-99m sulphide scan was not seen when the scan was repeated with L-selenomethionine ${ }^{75} \mathrm{Se}$, a phenomenon which has been reported with primary cancer of the liver. ${ }^{17}$

\section{RADIOLOGICAL INVESTIGATIONS}

Plain $x$-ray films of the abdomen were available in 37 cases. The liver was seen to be enlarged in all of these. Hepatic calcification was present in one instance. In five patients pulmonary metastases were evident on a plain film of the chest, and two of these also had bony metastases. Selective coeliac axis and superior mesenteric arteriography was performed in 20 patients. Displacement and stretching of the hepatic arteries or their branches indicating one or more space-occupying lesions in the liver was present in 12 patients. In three of these and a further six an irregular and bizarre pattern of the smaller branches of the hepatic arteries with a delay in the emptying of the capillaries (seen as a "blush") indicating a highly vascular tumour was shown. In two patients the only abnormality was tortuosity and pruning of the branches of the hepatic arteries, a picture seen in cirrhosis. The main hepatic artery was frequently widened, in contrast with the narrow hepatic artery seen in cirrhosis. Splenovenograms were available in nine instances. Six patients showed displacement or non-filling of the branches of the portal vein indicative of a mass in the liver. A further two subjects had a blocked portal vein, and in two others there was evidence of infiltration of the portal vein. Barium-meal examinations were performed in 16 of the subjects with ascites, and varices were shown in 14 .

\section{HISTOLOGY}

Percutaneous needle biopsies were performed in 57 patients, and $38(67 \%)$ were positive for the tumour. Wedge biopsy specimens taken at laparotomy were positive in a further nine.
In the remaining 28 cases a primary hepatic cancer was diagnosed at necropsy. In three instances the tumour was seen at necropsy to have invaded the hepatic veins, and in one of these tumour tissue had spread into the inferior vena cava and up into the right atrium. The portal vein was invaded and blocked by tumour and thrombosis in 13 patients. Of the 15 subjects in whom the portal vein was known to be obstructed (13 at necropsy and 2 on splenovenogram) 12 had ascites.

Fifty-two of the 65 patients $(80 \%)$ in whom this information was available had cirrhosis of the liver in association with the primary cancer. This was macronodular and of uncertain aetiology in 35, micronodular or mixed micronodular and macronodular and associated with a history of excessive alcohol consumption in 12, and associated with chronic active hepatitis in three and with haemochromatosis in two.

\section{Discussion}

Primary cancer of the liver is rare in most populations and produces no symptoms or signs pathognomonic of its presence. It is therefore not surprising that in many of the published series on this tumour the diagnosis was seldom made before death. ${ }^{1}$ s Clinical recognition depends primarily on an awareness of the condition. This is more likely to obtain in those geographical regions, such as the Orient and parts of Africa, where primary hepatic cancer is common. It can, however, be achieved elsewhere, as shown by the present series in which a definitive diagnosis was made during life in $63 \%$ of the patients and the condition suspected clinically in a further $20 \%$. The most common presenting complaints in these patients were abdominal pain or discomfort and weight loss. Pain was usually ill-defined, seldom severe, and had no diagnostic features. Weakness and malaise were also prominent though less frequent than in previous reviews. ${ }^{3}$ ?

Gastrointestinal symptoms such as nausea, vomiting, and loss of appetite were not infrequent but they are too non-specific to be of use in the diagnosis of primary hepatic cancer. Jaundice, haemorrhage from the gastrointestinal tract, and abdominal swelling were less common complaints. Primary cancer of the liver is often associated with hepatic cirrhosis, and it is often difficult to distinguish the signs and symptoms of the malignancy from those of the underlying lesion. One circumstance, however, which may alert the clinician to the possibility of a hepatic tumour is a sudden and unexplained deterioration in the clinical condition of a patient known to have cirrhosis or haemochromatosis; they may complain of abdominal pain, weight loss, or weakness, ascites may become a problem, or the liver may suddenly enlarge. This occurred in $24 \%$ of the present patients. A mode of presentation which was rare in our experience but has been reported in as many as $15 \%$ of some series ${ }^{6} 18$ was acute haemoperitoneum caused by the erosion of a vessel on the surface of the liver by the underlying primary cancer; the diagnosis is made at the ensuing laparotomy or at necropsy.

Physical findings obviously depend on the stage of the disease at which the patient presents. Early on the only abnormality may be a slightly or moderately enlarged liver. Frequently, however, the disease is advanced and the patients' general condition poor when they are first seen. The liver was almost invariably enlarged in these patients, but this finding is of limited value unless the enlargement is massive, the surface clearly irregular, or an obvious mass felt, as otherwise malignant infiltration may not be suspected. In this regard a most valuable sign is an arterial bruit heard over the liver. ${ }^{10}$ It was present in $25 \%$ of the present patients and, provided acute alcoholic hepatitis is excluded, ${ }^{10}$ provides an important clue to the diagnosis. A friction rub may also be heard over the liver, but this too is not pathognomonic of primary hepatic cancer as it has been reported with secondaries in the liver. ${ }^{20}{ }^{21}$

As in previous reviews the liver was seldom tender but may be extremely so. Ascites was often present but unless it is 
blood-stained or is shown to have a high protein content it is likely to be attributed to cirrhosis. Most patients were not jaundiced and when present it was usually mild. Fever was a feature in $24 \%$ of the cases, and though low-grade pyrexia may be present in patients with cirrhosis the finding of a persistently raised temperature should suggest the possibility of a hepatic neoplasm. Signs of chronic liver disease such as spider naevi and palmar erythema are probably due to the underlying cirrhosis rather than the tumour, though nine of the present patients without cirrhosis showed these signs. Rarely primary hepatic cancer may present as a Budd-Chiari syndrome owing to spread of the tumour into the hepatic veins. ${ }^{18}$ Portal vein thrombosis caused by the tumour may be responsible for ascites and bleeding from the oesophageal varices. In the absence of an obvious primary lesion it may be extremely difficult to distinguish primary cancer of the liver from hepatic secondaries.

As in previous reviews ${ }^{3} 4$ biochemical tests were of little value in the recognition of primary hepatic cancer; the changes in liver function tests were non-specific and in the main not distinct from those found in cirrhosis. A high serum alkaline phosphatase level with a normal or slightly raised serum bilirubin concentration, suggestive of a space-occupying or infiltrative lesion in the liver, ${ }^{22}$ was present in 24 patients. Subjects with this tumour occasionally develop profound hypoglycaemia ${ }^{23}$ such attacks occurred in three of the present patients and provided an important clue to the diagnosis. The finding of alpha-fetoprotein in the serum is virtually diagnostic of primary cancer of the liver. The frequency of positive results, however, has ranged from $80 \%$ in parts of Africa ${ }^{24}{ }^{25}$ to as little as $28 \%$ in America. ${ }^{\circ}$ In Britain the prevalence from this and a previous study ${ }^{26}$ is $38 \%$. At present this test offers the best chance to make an early diagnosis of primary cancer of the liver, and with more sensitive methods the number of positive results may be increased. ${ }^{27}$

Isotopic scanning of the liver is useful in establishing or confirming the presence of a space-occupying lesion, provided it is of reasonable size. This occurred in 30 out of 34 of these patients. There are, however, no features which are diagnostic of a primary tumour. Nor is it always possible to distinguish between hepatic and extrahepatic lesions. By doing an Lselenomethionine ${ }^{75} \mathrm{Se}$ scan in conjunction with a conventional scan it may be possible to obtain more definite evidence of a primary hepatic tumour; in a well-differentiated tumour uptake of L-selenomethionine ${ }^{75} \mathrm{Se}$ by the malignant tissue is similar to that by the rest of the liver, thus obliterating the filling defect on a technetium scan. ${ }^{17}$. The technique may fail, however, if the tumour is very vascular or necrotic, and falsepositive results have also been reported. ${ }^{17}$ Hepatic scanning is also useful in localizing the tumour for biopsy purposes and determining its extent if resection is contemplated.

Hepatic angiography may also be extremely valuable. Selective coeliac axis and superior mesenteric arteriography may show the presence of masses in the liver by displacement and stretching of the hepatic arteries or their branches. More important is finding an irregular and bizarre pattern of the smaller branches of the hepatic arteries with a delay in emptying of the capillaries, which is characteristic of a highly vascular tumour. As primary hepatic tumours are usually very vascular, and as these signs are less common with hepatic metastases, ${ }^{28}$ this finding favours a primary tumour. Splenic venography may be used to show the presence of space-occupying lesions in the liver by displacement or non-filling of the branches of the portal vein. Visualization of the portal branches can be improved and smaller masses detected by injection of the dye through the catheterized umbilical vein. ${ }^{29}$. Portal venography may also show infiltration of the portal, vein by tumour or portal vein"thrombosis. Neither arteriography nor venography is invariably positive, however, and they are more likely to be of value in the more advanced stages of the disease.

Confirmation of the diagnosis is histological. This can frequently and conveniently be achieved by percutaneous needle biopsy, especially if a liver scan is available to localize the lesion. Needle biopsies were positive in 38 out of 57 of the present patients. The diagnosis may also be confirmed by wedge biopsy at exploratory laparotomy. In 28 patients in the present series histological confirmation of the tumour was obtained only at necropsy.

Unfortunately, the disease runs a short course and has almost always been far advanced when the diagnosis was made, so that surgical resection has rarely been possible and other forms of treatment have been almost entirely unsuccessful. With the possible exception of the finding of alpha-fetoprotein in the serum the diagnostic techniques currently in use are of value only when the disease is well advanced. If the prognosis of these patients is to be improved by early diagnosis attention will have to be directed towards finding more sensitive and more specific indices of the presence of the tumour.

One of us (M.C.K.) is in receipt of a Wellcome research grant, and another (H.A.D.S.) a grant from C.A.P.E.S. in Brazil. Financial support for this study was provided by the Johnston Trust. We are grateful to our colleagues in the departments of radiology and medical physics for their assistance.

\section{References}

1 Wilbur, D. L., Wood, D. A., and Willett, F. M., Annals of Internal Medicine, 1944, 20, 453.

${ }^{2}$ Holley, H. L., and Pierson, G., American Fournal of Medicine, 1948, 5,561 .

${ }^{3}$ Moseley, R. V., Surgery, 1967, 61, 674.

4 Bersohn, I., Purves, L. R., and Geddes, E. W., South African Medical fournal, 1969, 43, 1219.

5 Hoyne, R. M., and Kernohan, J. W., Archives of Internal Medicine, 1947, 79, 532 .

6 Berman, C., Primary Carcinoma of the Liver. London, Lewis, 1951.

7 MacDonald, R. A., Archives of Internal Medicine, 1957, 99, 266

8 Tatarinov, Y., Voprosỹ Meditsinskoi Khimiz, 1964, 10, 90.

9 Alpert, M. E., Clinical Research, 1969, 17, 461. 10 Abelev, G. I., Assecritova, I. V., Kraevsky, N. A., and Perova, S. D.,
International fournal of Cancer, 1967, 2, 551.

11 Houstek, J., Masopust, J., Kithier, K., and Radl, J., fournal of Pediatrics, 1968, 72, 186.

12 Mawas, C., et al., International fournal of Cancer, 1969, 4, 76.

13 Bourreile, J., Metayer, P., Sauger, F., Matray, F., and Fondimore, A., Presse Médicale, 1970, 78, 1277.

14 Geffroy, Y., et al., Presse Médicale, 1970, 78, 1107.

15 Foy, H., Kondi, A., Parker, A. M., Stanley, R., and Venning, C. D., Lancet, 1970, 1, 1336.

${ }_{16}$ Alpert, M. E., and Seeler, R. A., Fournal of Pediatrics, 1970, 77, 1058.

17 Rake, M. O., Eddleston, A., Pagaitsos, S., Williams, R., and Osborn, S. B., British fournal of Radiology, 1970, 43, 830.

18 Benner, E. J., and Labby, D., Annals of Internal Medicine, 1961, 54, 620.

19 Benner, E. J., and Labby, D., Annals of Internal Medicine, 1961,

20 Fenster, L. F., and Klatskin, G., American fournal of Medicine, 1961, $31,238$.

21 Simpson, J. A., American fournal of Digestive Diseases, 1971, 16, 39.

22 Ross, R. S., Iber, F. L., and Harvey, A. M., American fournal of Medicine, 1956, 31, 850 .

${ }^{23}$ Klein, H., and Klein, S. P., Archives of Internal Medicine, 1959, 103, 273.

24 Purves, L. R., MacNab, M., and Bersohn, I., South African Medical Fournal, 1968, 42, 1138 .

25 Uriel, J., et al., Presse Médicale, 1968, 76, 1415.

${ }^{26}$ Foli, A. K., Sherlock, S., and Adinolfi, M., Lancet, 1969, 2, 1267.

27 Purves, L.' R., Bersohn, I., and Geddes, E. W., Cancer (Philadelphia), 1970, 25, 1261 .

${ }^{28}$ Reuter, S. R., Redman, H. C., and Siders, D. B., Radiology, 1970, 94, 89.

${ }_{29}$ Man, B., Krans, L., and Pikielny, S., Clinical Radiology, 1971, 32, 350. 